\title{
Polarization tunable selective polariton generator
}

\author{
N. Sedoglavich, ${ }^{1, a)}$ R. Künnemeyer, ${ }^{1}$ and J. C. Sharpe ${ }^{2}$ \\ ${ }^{1}$ Department of Engineering, The University of Waikato, Hamilton 3216, New Zealand \\ ${ }^{2}$ The New Zealand Institute for Plant and Food Research Limited, Hamilton, New Zealand
}

(Received 8 December 2008; accepted 22 February 2009; published online 12 March 2009)

\begin{abstract}
A selective polariton generator (SPG) design, based on surface plasmon antennae principles, is demonstrated to provide a selective light transmission peak. The polarization-sensitive structure selectively generates and transports polaritons of a desired wavelength through a circular subwavelength aperture. By varying the SPG structure around a central nanohole, we are able to control the peak optical transmission wavelengths via the polarization state of the incident photons. We find good agreement between simulations and experimental results. (C) 2009 American Institute of Physics. [DOI: 10.1063/1.3099040]
\end{abstract}

According to the diffraction theory, apertures smaller than the wavelength of light have very low transmission. As proposed by Bethe, ${ }^{1}$ the transmission, normalized to the area of the hole, scales as $(D / \lambda)^{4}$, where $D$ is the hole diameter and $\lambda$ is the wavelength. However, significant improvements have been made since the report of Ebbesen et $a l^{2}$ on extraordinary optical transmission through nanohole arrays in metal films, the results of which challenge Bethe's work. ${ }^{1}$ Surface waves such as surface plasmons, which are electromagnetic waves trapped at a metallic surface through their interaction with free electrons of the metal, play a principal role in this phenomenon. Surface plasmon waves result under special momentum matching circumstances where energy is transferred from a photon into an oscillating electron wave. ${ }^{3}$ The understanding and interaction of nanogeometries on metal-dielectric interfaces has led to unique nanophotonic materials and devices by exploiting the excitation and localization of surface plasmon polariton modes. ${ }^{4}$ Nanofabrication techniques, such as focused ion beam (FIB) milling, permit the fabrication of structures with complex surface wave dynamics. ${ }^{5,6}$

For a periodically modulated interface between a metal and a dielectric with period $\Lambda$, the surface component of the wave vector $2 \pi / \lambda_{0}$ of the incident light can increase (or decrease) by integral multiples of the grating wave vector $2 \pi / \Lambda .^{7}$ If this increase matches that of surface plasmon waves in the metal, it can couple to and excite them. Therefore, momentum can be matched using metal diffraction gratings. ${ }^{8}$ The wave vector of the surface plasmon wave $k_{\mathrm{SP}}$ is given by

$$
k_{\mathrm{SP}}=\frac{2 \pi}{\lambda_{0}} \sqrt{\frac{\varepsilon_{\mathrm{mr}} n_{D}^{2}}{\varepsilon_{\mathrm{mr}}+n_{D}^{2}}},
$$

where $2 \pi / \lambda_{0}$ is the free space wave number, $\varepsilon_{\mathrm{mr}}$ is the real permittivity component of the metal, and $n_{D}$ is the refractive index of the dielectric. The wave vector of the diffracted wave $k_{\mathrm{S}}$ is calculated using

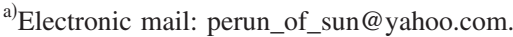

$$
k_{\mathrm{S}}=\frac{2 \pi}{\lambda_{0}} n_{D} \sin \theta_{0}+m \frac{2 \pi}{\Lambda},
$$

where $\theta_{0}$ is the angle of incidence, $\lambda_{0}$ the laser wavelength in vacuum, and $m$ is an integer $(0, \pm 1, \pm 2 \ldots)$ indicating the order of diffraction. As the grating structures contribute to the momentum, it can be designed so that $k_{\mathrm{S}}$ equals $k_{\mathrm{SP}}$, matching the incident light with surface plasmon waves. Therefore, a single aperture surrounded by a periodic corrugation in the metal surface will also display surface plasmonenhanced transmission.

Previous studies on annular surface plasmon structures have investigated the effects of variables such as corrugation period and depth, ${ }^{9}$ while their susceptibility to incident light polarization has been overlooked. In our previous work, we have reported on surface plasmon polariton structures with specific discrete polarization and wavelength selectivity. ${ }^{10}$ These selective polariton generators (SPGs) have been simulated, fabricated, and tested for optical transmission, gated switching, and polarization-selective properties. The effect of the number of corrugations on the grating performance has also been studied. It was found that a small increase of around $7 \%$ in transmission intensity was achieved by adding a fifth resonant corrugation ring. ${ }^{10}$ This indicates an approaching saturation point, limiting the number of resonant corrugation rings by the rate of decay of traveling surface plasmon polaritons. ${ }^{10}$ Also, there was no significant change in the peak wavelength position. Therefore, four corrugations were found to be a good balance in the structure's size, while still maintaining optimal performance.

Here we present a polarization tunable SPG structure that has been designed to permit continuous tuning of its wavelength dependant transmission peak by the angle of the incident, linear polarized light. The design is based on a nanohole surrounded by a grating geometry where the grating period varies from 570 to $690 \mathrm{~nm}$ as the angle around the nanohole changes from the horizontal to vertical axis. It is the intention that light with a particular polarization angle only interacts with the corresponding corrugation periodicity. The range of corrugation periodicity was spread over $90^{\circ}$, symmetrically encircling the central nanohole. The continuously tunable SPG structures were designed to have a linear relationship between the change in polarization angle and the change in the corrugation period. The elliptical geometry 


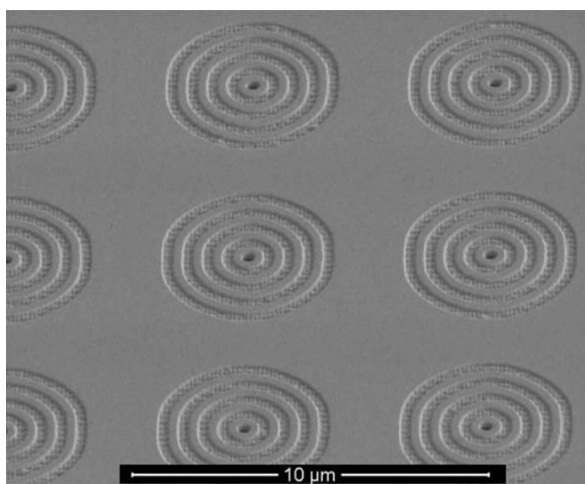

FIG. 1. Scanning electron microscope image, taken at $52^{\circ}$ to the substrate surface, of a linearly tunable SPG fabricated using FIB milling. Corrugation periods along the horizontal and vertical axes are 570 and $690 \mathrm{~nm}$, respectively.

was found not to exhibit a linear relationship between the change in the polarization angle and the change in the corrugation period. Therefore, the new geometry was designed to provide the linear transition. The radius of each corrugation is related to the structures periodicity, which was defined by

$$
\Lambda=\Lambda_{i}+\frac{2 \theta}{\pi}\left(\Lambda_{f}-\Lambda_{i}\right)
$$

where the current periodicity $\Lambda$ depends on $\Lambda_{i}$ and $\Lambda_{f}$ the initial and final periodicity, respectively, and the polarization angle $\theta$. The derivative of Eq. (3) results in a linear relationship between the change in periodicity and the change in the polarization angle.

The devices were fabricated on gold-coated glass substrates $\left(200 \mathrm{~nm} \mathrm{Au}\right.$ film on $4 \mathrm{~nm}$ Ti on $1 \times 10 \times 10 \mathrm{~mm}^{3}$ glass, SSENS, Netherlands) using FIB milling (dual-beam xT Nova NanoLab, Ga ion source, $30 \mathrm{kV}$ source) at a beam current of $100 \mathrm{pA}$. The resulting corrugation depth was $80-90 \mathrm{~nm}$. A scanning electron microscope image of the linearly tunable SPG array is shown in Fig. 1 , taken at $52^{\circ}$ to the substrate surface.

Experimental measurements of spectral transmission of the substrate were performed using a purpose-built microscope. Samples were illuminated by a broadband halogen source (AvaLight-Hal-S, Avantes, Netherlands) through a $400 \mu \mathrm{m}$ core diameter fiber [0.22 numerical aperture (NA)]. A $100 \times$ objective lens (ELWD, Nikon, Japan), with an NA of 0.8 and extended near-infrared transmission, was used to collect the transmitted light. The magnified image was projected onto a $400 \mu \mathrm{m}$ core diameter optical fiber (0.12 NA) coupled to a charge-coupled device (CCD) spectrometer (Ocean Optics, Inc., Dunedin, FL) or onto a CCD camera to facilitate substrate alignment. The transmission spectra were integrated for $5 \mathrm{~s}$ and averaged over three samples. They were then corrected for dark current, background light, and normalized to the spectral envelope of the halogen lamp. A polarizer was mounted on a rotation stage in the detection path to provide analyses at different polarization angles. Simulations support the concept that there is no change in polarization between the incident light and the transmitted light. Because of the magnification of the setup, individual SPG elements could be studied.

The experimentally obtained spectral and polarization characteristics of the SPG structure are shown in Fig. 2. The

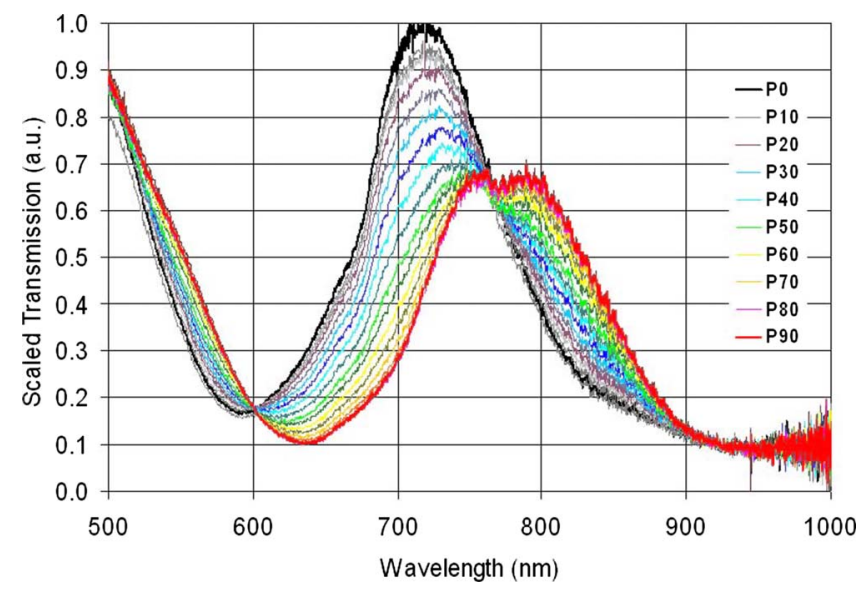

FIG. 2. (Color online) Experimental results of the linearly tunable SPG for light polarization angles from $0^{\circ}$ to $90^{\circ}$.

data was recorded at $5^{\circ}$ intervals over a $360^{\circ}$ range of polarization angles. The figure presents a subset where the light polarization angle was varied over a range of $90^{\circ}$. At a polarization angle of $0^{\circ}$, parallel to the $570 \mathrm{~nm}$ corrugation, horizontal axis, the transmission curve peaks at $718 \mathrm{~nm}$. As the polarization angle is increased, the transmission peak gradually shifts to longer wavelengths. When the angle is increased past $90^{\circ}$, i.e., parallel to the $690 \mathrm{~nm}$ corrugation axis, the transmission curve starts to shift back to the blue wavelength range. The cycle repeats as the polarization angle is rotated through a full $360^{\circ}$.

The experimental results demonstrate the structure's tunable characteristics, with the transmission peak varying from 718 to $758 \mathrm{~nm}$. However, a minor secondary peak develops as the polarization angle approaches $90^{\circ}$, resulting in a dual peak transmission curve.

The structure was modeled using finite-difference timedomain software (FDTD) (Lumerical Solutions Inc., Vancouver, Canada). The model collected transmitted light within a cone of $15^{\circ}$. As it was not possible to simulate continuously varying periodicity, the FDTD model was an approximation of the true structure, with the corrugation periods discretized into $10^{\circ}$ steps (Fig. 3). The corrugation depth was set to $60 \mathrm{~nm}$. The figure shows a particular simulation where the

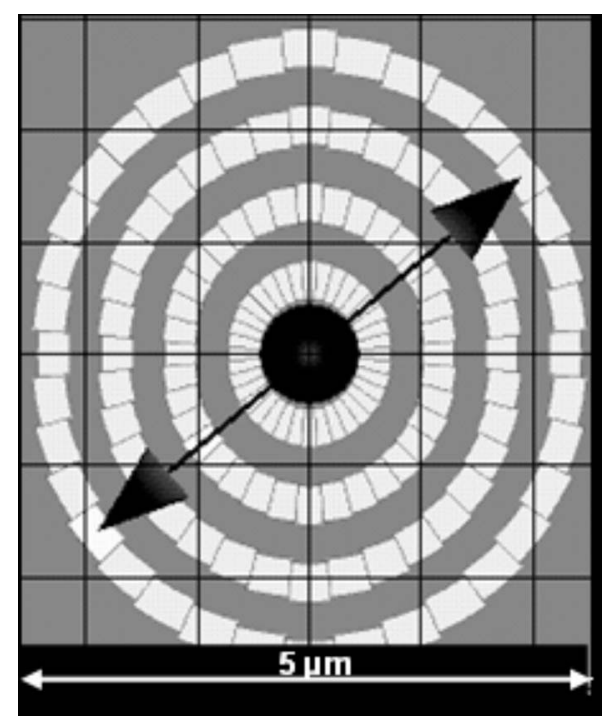

FIG. 3. FDTD discretized model of the linearly tunable SPG structure. 


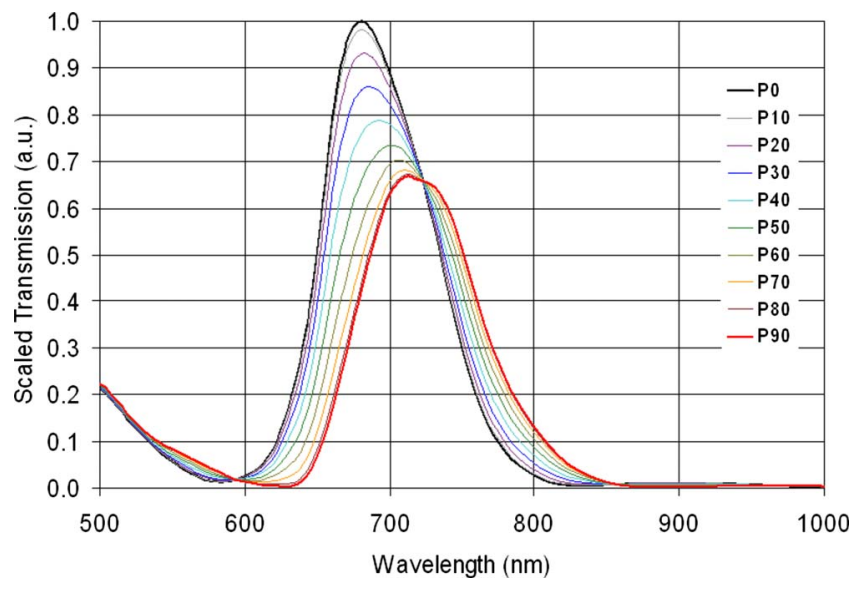

FIG. 4. (Color online) Simulated results of the linearly tunable SPG.

incident light polarization was set to $40^{\circ}$ from the $570 \mathrm{~nm}$ corrugation axis.

The simulated spectral and polarization characteristics of the tunable SPG structure are shown in Fig. 4. The simulation was run at $10^{\circ}$ polarization angle intervals starting at $0^{\circ}$. At $0^{\circ}$, the transmission curve peaks at $680 \mathrm{~nm}$, redshifting as the polarization is increased toward $90^{\circ}$. The simulated data exhibits similar spectral and polarization characteristics as seen in the experimental results. The most noticeable differences are the transmission peak, which is blueshifted by about $40 \mathrm{~nm}$ and the smaller secondary peak in the simulated data.

The extraordinary optical transmission for the structure was calculated to be 17.2 and 12.1 for the polarization angles of $0^{\circ}$ and $90^{\circ}$, respectively. The extraordinary optical transmission is defined as the ratio of the output to input power flux, the output power over the nanohole area divided by the power over the illuminated surface area.

Figure 5 presents the wavelength change in the left transmission peak as the light polarization angle is varied through $90^{\circ}$. A second $x$-axis is shown to illustrate the expected corrugation period at each polarization angle. In the middle region, between $20^{\circ}$ and $70^{\circ}$, the peak transmission wavelength is linearly dependent on the polarization angle in the simulations, while at the extremes the rate of change decays away. Similar transmission behavior is shown in the experimental results. Both the experimental and simulated data exhibit the same spectral shift in $40 \mathrm{~nm}$ over the full range of polarization angles. The error bars in the experimental results correspond to the readout error, $\pm 1^{\circ}$ for the polar-

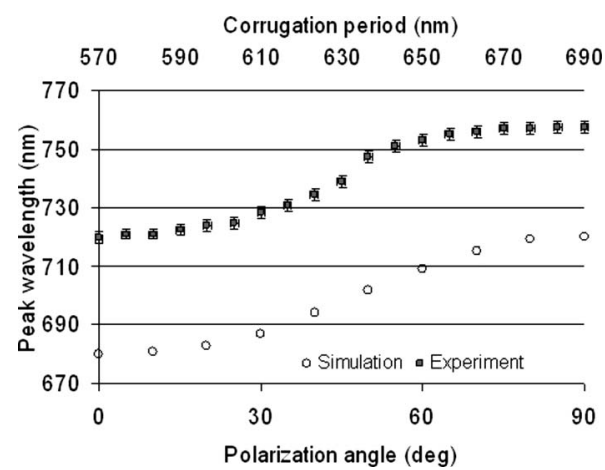

FIG. 5. Comparison of the experimental and the simulated results, tracking the left transmission peak

ization angle and $\pm 2 \mathrm{~nm}$ for the peak transmission wavelength.

In conclusion, we have developed, fabricated, and characterized a surface plasmon polariton structure, which can, by controlling polarization as a tuning variable, selectively generate and transport polaritons of a desired wavelength. This SPG device combines a tunable plasmon resonator and a nanohole. By specifying geometry and orientation we can control the structure's operational characteristics, as demonstrated by close agreement between the experimental results and FDTD simulated data. We anticipate, due to the previously described characteristics, that SPGs have the potential for development and application in areas ranging from polariton generators, and multistate nanophotonic switching and devices, to tunable spectroscopic elements and biosensors.

We would like to acknowledge Top Achievers Doctoral funding from the Tertiary Education Commission of New Zealand and Capability Funding from Plant and Food Research Institute Limited.

${ }^{1}$ H. A. Bethe, Phys. Rev. 66, 163 (1944).

${ }^{2}$ T. W. Ebbesen, H. J. Lezec, H. F. Ghaemi, T. Thio, and P. A. Wolff, Nature (London) 391, 667 (1998).

${ }^{3}$ J. Homola, S. S. Yee, and G. Gauglitz, Sens. Actuators B 54, 3 (1999).

${ }^{4}$ W. L. Barnes, A. Dereux, and T. W. Ebbesen, Nature (London) 424, 824 (2003).

${ }^{5}$ A. Degiron, H. J. Lezec, N. Yamamoto, and T. W. Ebbesen, Opt. Commun. 239, 61 (2004)

${ }^{6}$ F. J. Garcia-Vidal, H. J. Lezec, T. W. Ebbesen, and L. Martin-Moreno, Phys. Rev. Lett. 90, 2139012003.

${ }^{7}$ B. K. Singh and A. C. Hillier, Anal. Chem. 78, 2009 (2006).

${ }^{8}$ E. Fontana, Appl. Opt. 43, 79 (2004).

${ }^{9}$ K. L. Shuford, M. A. Ratner, S. K. Gray, and G. C. Schatz, Appl. Phys. B: Lasers Opt. 84, 11 (2006).

${ }^{10}$ N. Sedoglavich, J. Sharpe, R. Künnemeyer, and S. Rubanov, Opt. Express 16, 5832 (2008). 